\title{
CARDIOPULMONARY EXERCISE TESTING: UTILITY IN RESEARCH AND PATIENT CARE
}

\author{
Elina $R A^{\prime}$, Husain $R^{\prime}$ and Lang $C C^{2}$ \\ ' Department of Physiology, Faculty of Medicine, University of Malaya, 50603 Kuala Lumpur, Malaysia \\ 2 Department of Cardiology, Ninewells Hospital and Medical School, Dundee, United Kingdom
}

\begin{abstract}
Cardiopulmonary exercise testing is a non-invasive physiological test which incorporates the conventional method of exercise stress test with a more advanced breath-to-breath ventilatory analysis. The physiological parameters obtained from the test help to illustrate the cardiovascular, respiratory and metabolic responses to physical exertion. Individual's functional capacity and aerobic fitness is reflected by the value of maximal oxygen consumption $\left(\mathrm{VO}_{2}\right.$ max) obtained from the cardiopulmonary exercise test. This non-invasive and sophisticated test is regarded as a valuable assessment tool in research and clinical practice. Cardiopulmonary exercise test has been extensively utilized to define the mechanisms of exercise intolerance in various clinical disorders, to evaluate responses to therapy and indicate disease prognosis. Emerging data obtained from the use of the cardiopulmonary exercise testing in the research field, has led to its extensive clinical usage. It is now utilized as an integral part of the patients' clinical evaluation in the field of respiratory and cardiovascular medicine, sports medicine, surgery as well as occupational and rehabilitative medicine. It has a clinical role in assessing patient's functional capacity, monitoring disease progression and response to therapy, predicting prognosis, and perioperative morbidity and mortality, as well as constructing and monitoring training and rehabilitative programs. This article aims to give an overview of the physiological profiles obtained from cardiopulmonary exercise testing, its methodological aspects, as well as its utility in research and clinical practice. (JUMMEC 2003-2005; 8: 9-22)
\end{abstract}

KEYWORDS: Cardiopulmonary, exercise, physiology, respiratory medicine, oxygen consumption

\section{Introduction}

Exercise is the most common form of physiological stress encountered by the body. It requires a coordinated interaction of virtually all body systems to effectively adapt to the physiological and metabolic demands incurred.The cardiopulmonary system is one of the crucial systems in the body which copes with the major demands of exercise, ensuring adequate oxygen delivery to the working muscles. Exercise test has traditionally been regarded as a valuable tool to evaluate cardiac perfusion and function under controlled conditions, due to its ability to detect cardiac dysfunction under stress, which may not necessarily be present at rest. Recently, the incorporation of ventilatory gas analysis into the conventional method of exercise testing has further expanded its utility in research and clinical field. Formal cardiopulmonary exercise testing is a non-invasive and sophisticated physiologic testing technique, which includes the recording of the exercise ECG, heart rate and blood pressure responses to exercise, minute ventilation, and allows calculation of the subject's maximal oxygen consumption. It provides a comprehensive functional assessment, that helps to define the relative contribution of various human physiological systems (cardiovascular, pulmonary and muscle metabolism) in determining exercise performance.

At the early phase of exercise, there is a gradual increase in oxidation of substrate such as carbohydrate and fat for regeneration of high energy in the form of

Correspondence:

Dr Raja Elina Afzan binti Raja Ahmad

Department of Physiology

Faculty of Medicine, University of Malaya

50603 Kuala Lumpur.

Tel: 603-7967 4742

Fax: 603-7967 4775

Email: rajaelinaafzan@um.edu.my 
Adenosine Triphosphate (ATP), to sustain muscular contractions. The increasing metabolic requirements of the cells under stress can essentially be met by effective adaptations of the central (cardiopulmonary) and peripheral systems (skeletal muscle cellular and metabolic responses) in the body. The respiratory system provides enhanced ventilation and gas exchange during exercise. The cardiovascular system adapts by increasing the cardiac output to ensure effective systemic oxygen transportation. In addition, there is an increase in oxygen extraction by the working muscles, reflecting adaptation of the peripheral system to this state of physiological stress. Disturbance in any one of these systems will cause considerable changes in the adaptations to an acute episode of exercise, and this can be quantified during cardiopulmonary exercise test. Hence, the cardiopulmonary exercise test allows for an integrated assessment of the cardiovascular response, respiratory mechanics, gas exchange process and metabolic responses to the stress of physical exertion.

\section{Physiological basis of cardiopulmonary exercise testing}

During exercise, both oxygen delivery and tissues oxygen extraction rise. The increase in oxygen delivery during exercise is primarily achieved by increasing the cardiac output (four to five folds) (I). There is a linear increase in both oxygen consumption and cardiac output with increasing workload, until an individual maximum value is reached. This oxygen consumption plateau, termed maximal oxygen consumption $\left(\mathrm{VO}_{2} \mathrm{max}\right)$, is the hallmark of cardiopulmonary exercise testing, representing a marker of exercise capacity or cardiopulmonary fitness. $\mathrm{VO}_{2} \mathrm{max}$ is defined as the maximum amount of oxygen a person can take in the form of inspired air while performing dynamic exercise involving a large muscle group (2). It represents the amount of oxygen transported and utilized in the cellular metabolism, as reflected by the Fick principle: $\left(\mathrm{VO}_{2}\right.$ max $=$ maximal cardiac output $\left.(\mathrm{CO})\right)$ $x$ maximal arteriovenous oxygen difference $\left(a-\mathrm{vO}_{2}\right.$ diff). Because the arterial-venous oxygen difference is generally considered to widen by a relatively fixed amount during exercise, and $\mathrm{VO}_{2} \max$ is typically achieved by exercise that involves at least half of the total body musculature (that is, not limited to a focal area), it is generally believed that $\mathrm{VO}_{2} \mathrm{max}$ is primarily limited by cardiac output rather than the peripheral oxygen extraction (3). Hence, $\mathrm{VO}_{2} \max$ defines the upper limit of cardiac function during aerobic exercise, and is therefore, utilized as an objective measure of cardiorespiratory fitness during cardiopulmonary exercise testing. Apart from differences in cardiopulmonary responses to exercise, factors which are known to influence the individual variation of $\mathrm{VO}_{2} \max$ include age, gender, individual fitness or conditioning status, type of exercise being performed, the presence of various diseases, medications, genetic factor, and environment where the particular exercise is being performed (2).

Maximal oxygen consumption $\left(\mathrm{VO}_{2} \max \right)$ is primarily influenced by the determinants of cardiac output. Abnormality in $\mathrm{VO}_{2}$ max may reflect either inability to increase heart rate or failure to augment stroke volume during exercise. During exercise, stroke volume increases up to approximately $50 \%$ to $60 \%$ of an individual's maximal exercise capacity (up to 100$200 \mathrm{ml} /$ beat in well-trained subjects), after which, increases in cardiac output are caused by further increases in heart rate (3). However, in untrained subjects, cardiac output is increased almost entirely by an increase in heart rate. Physical training will improve augmentation of stroke volume, which results in better cardiovascular endurance during exercise. Cardiopulmonary assessment has therefore been utilized as a functional assessment tool that forms the basis for exercise prescription, to enhance athlete's performance or for cardiac rehabilitation.

The most common respiratory parameters assessed during exercise are changes in total minute ventilation $\left(V_{E}\right)$, tidal volume $\left(V_{T}\right)$ and respiratory rate $(R R)$. In order to have effective consumption of oxygen during exercise, augmentation of ventilation is required to complement the increase in cardiac output. Increases in minute ventilation during exercise are initially achieved by increasing both the tidal volume and respiratory rate. Ventilatory limitation to exercise can be established by evaluating the individual's ventilatory reserve, defined as the difference between maximum voluntary ventilation (MVV) and maximum spontaneous ventilation (VE max) that is, $V R=M V V-V E$ max. This parameter reflects the ability of ventilation to approach maximum volume that can be respired per minute (MVV), and is often reduced in patients with pulmonary diseases. Other ventilatory parameters which are often obtained during cardiopulmonary exercise testing and are indicative of gas exchange efficiency include ventilatory equivalents for $\mathrm{O}_{2}$ $\left(\mathrm{V}_{\mathrm{E}} / \mathrm{V}_{\mathrm{O} 2}\right)$ and $\mathrm{CO}_{2}\left(\mathrm{~V}_{\mathrm{E}} / \mathrm{V}_{\mathrm{CO} 2}\right)$, as well as the ratio of dead space volume to tidal volume $\left(\mathrm{V}_{\mathrm{D}} / \mathrm{V}_{\mathrm{T}}\right)$. In addition, the measurement of differences in oxygen partial pressure between alveoli and pulmonary capillaries $\left(\mathrm{P}(\mathrm{A}-\mathrm{a}) \mathrm{O}_{2}\right)$ is also helpful in ascertaining the cause of hypoxaemia during exercise.

The metabolic events during cardiopulmonary exercise can be evaluated by measuring respiratory exchange ratio (RER) and anaerobic threshold (AT). The ratio of oxygen consumption and carbon dioxide 
output $\left(\mathrm{VO}_{2} / \mathrm{VCO}_{2}\right)$ is called the respiratory exchange ratio (RER), which can be used as a rough index of metabolic activity. Anaerobic threshold (AT) is considered an estimator of the onset of metabolic acidosis, caused predominantly by increased rate of rise in arterial lactate during exercise. It is referred to the value of $\mathrm{VO}_{2}$ at the onset of anaerobic metabolism, and is commonly expressed as a percentage from the $\mathrm{VO}_{2}$ max predicted value for each individual. In normal sedentary individuals, the AT occurs at about $40 \%-60 \%$ of predicted $\mathrm{VO}_{2} \max$ (4), with higher value in endurance-trained individuals. Values below $40 \%$ of predicted $\mathrm{VO}_{2}$ max may indicate cardiac, pulmonary or other limitations in oxygen supply to the tissues, or other underlying cellular mitochondrial activity (for example, mitochondrial myopathies).

\section{Cardiopulmonary exercise mode and protocol selection}

Dynamic exercise involving large muscle groups is preferred for clinical exercise testing, since it initiates a more appropriate increase in cardiac output and oxygen exchange. Bicycle ergometer and the motorized treadmill are now the most commonly utilized dynamic exercise devices. Bicycle ergometer has the advantage of being relatively cheap and takes up less space compared to the treadmill. However, it requires the subject's cooperation to maintain the pedaling speed at the desired level, usually about $60 \mathrm{rpm}$, to ensure achievement of the desired workload. In addition, some subjects are not familiar with bicycling, as compared to walking exercise. Cycle ergometry has also been shown to result in $10 \%$ to $20 \%$ lower value of maximal oxygen consumption as compared to treadmill exercise (5). Thus, the treadmill is a generally more common dynamic testing modality, except in situations where the subject suffers from gait instability, or when simultaneous cardiac imaging is planned. Arm ergometry is an alternative method of exercise testing for patients with vascular, neurologic or orthopaedic conditions, who cannot perform leg exercises. However, at maximal effort, the extent of physiological responses in arm exercise is generally less than leg exercise due to recruitment of smaller muscle bulk.

The selection of appropriate workload protocol during cardiopulmonary test is of critical importance, as it has a considerable influence on the interpretation of physiologic parameters obtained during the exercise test. With each protocol, the measurement of workload or performance is expressed in basal metabolic equivalents (METs). One METs corresponds to the resting oxygen consumption rate of $3.5 \mathrm{~mL}$ $\mathrm{O}_{2} / \mathrm{kg} / \mathrm{min}^{2}$. Before selecting the exercise protocol, the investigator must first decide whether to conduct the exercise test at a maximal or submaximal level.
Patient's true maximal effort and functional capacity can be better evaluated from a maximal treadmill walking test. The protocol for maximal dynamic exercise testing can be classified according to the manner in which the workload is applied (that is, incremental or constant exercise workload). The traditional Bruce treadmill protocol is the most commonly used exercise protocol used in routine clinical setting (6). However, it involves vigorous exercise, commencing at five METs of energy, and increases both speed and gradient with each 3-minute stage. Because of its large unequal workload increment between stages and rapid exertion level, the protocol has been associated with overestimation of the subject's exercise capacity $\left(\mathrm{VO}_{2}\right.$ max), particularly for patients with cardiac disease (7). Moderate increment in treadmill elevation at a constant speed, are preferable protocols to accurately estimate the subjects maximal oxygen consumption (2). Recently, the 'Ramp' protocol which involves small increments in work rate at 30 to 60 -second interval, has been shown to accurately determine patients' functional capacity during exercise (8). Table I summarises the physiological profiles obtained and Table 2 illustrates some of the exercise protocols which can be applied during cardiopulmonary exercise test. Regardless of the specific protocol selected, it should be optimized to reach the individual's symptom-limited maximal effort, with exercise duration between 10 to 12 minutes. Shorter exercise duration may disrupt the linear relationship between $\mathrm{VO}_{2} \max$ and work rate, whereas longer duration of more than 12 minutes may cause the subjects to terminate the exercise because of muscle fatigue, before reaching their true maximal effort.

Although maximal testing provides a more accurate determination of aerobic capacity, submaximal exercise testing may be desirable in certain situations which preclude strenuous physical activity. This includes predischarge cardiac evaluation after myocardial infarction, patients with dangerous dysrhythmias, assessment of frail elderly subjects who are unaccustomed with vigorous exercise, and field testing of a large number of subjects, particularly in the absence of clinical supervision. In the recent years, the six or 12-minute walk have been widely utilized to determine patient's submaximal functional capacity (9). Such test modality is found to be simpler, safe, very inexpensive and applicable to simulate performance of daily activities. The distance covered during 6-minute walk test was found to be a good prognostic indicator of patients with heart failure (9). It has also been employed as an assessment tool to evaluate therapeutic intervention, particularly pacemaker therapy, in heart failure patients $(I 0, I I)$. 
Table I. Summary of the physiological profiles obtained from cardiopulmonary exercise test

\begin{tabular}{ll}
\hline Physiological Variables & System Assessed \\
\hline - $\mathrm{VO}_{2}$ max & \\
- $\mathrm{O}_{2}$ pulse & \\
- Heart rate & Assessment of cardiovascular response during exercise \\
- $\mathrm{ECG}$ & \\
- $\mathrm{V}_{\mathrm{E}}$ & \\
- $\mathrm{V}_{\mathrm{T}}$ & \\
- RR & \\
- $\mathrm{VR}$ & Assessment of respiratory mechanics during exercise \\
- $\mathrm{MVV}$ & \\
- $\mathrm{V}_{\mathrm{E}} / \mathrm{VO}_{2}$ & \\
- $\mathrm{V}_{\mathrm{E}} / \mathrm{VO}_{2}$ & \\
- $\mathrm{V}_{\mathrm{D}} / \mathrm{V}_{\mathrm{T}}$ & \\
$-\mathrm{RQ}$ & Assessment of gas exchange process during exercise \\
- $\mathrm{RT}$ & \\
\hline
\end{tabular}

$\mathrm{VO}_{2} \max =$ maximal oxygen consumption; $\mathrm{O}_{2}=$ oxygen; $\mathrm{ECG}=$ electrocardiography; $\mathrm{VE}=$ total minute ventilation;

$\mathrm{VT}=$ tidal volume; $\mathrm{RR}=$ respiratory rate; $\mathrm{VR}$ = ventilatory reserve; $\mathrm{MVV}=$ maximum voluntary ventilation;

$\mathrm{VO}_{2}=$ oxygen consumption; $\mathrm{VCO}_{2}=$ carbon dioxide production; $\mathrm{VD}=$ volume of physiological dead space;

$\mathrm{RQ}=$ respiratory quotient; $\mathrm{AT}=$ anaerobic threshold.

Table 2. Summary of various exercise protocols during cardiopulmonary exercise testing

\section{Treadmill exercise protocols}

Bruce Protocol

Initial speed $1.7 \mathrm{mph}$ and $10 \%$ slope; increments of $0.8 \mathrm{mph}$ and $2 \%$ slope every 3 minutes

Balke Protocol Constant speed and increase of slope by $1 \%$ per minute

Naughton Protocol Initial speed $3.2 \mathrm{~km} / \mathrm{h}$ with $3.5 \%$ slope every 3 minutes

\section{Bicycle ergometer protocols}

Incremental exercise test $\quad 60 \mathrm{rpm}$; increase 5-25 watt/min; planned test duration 6- 12 minutes

Ramp Protocol Continuously increasing exercise; increase at a one-second interval

\section{Research application of cardiopulmonary exercise test}

Cardiopulmonary exercise test is regarded as an important research tool, which can be utilized as a mean of objective assessment of functional capacity and fitness level, in virtually any field of research, involving healthy and medically unfit individuals. In the area of clinical research, cardiopulmonary exercise test has been predominantly utilized to define the mechanism of exercise intolerance in various clinical disorders, as well as to objectively evaluate the efficacy of therapeutic interventions. Comprehensive cardio- pulmonary data obtained from the test, provides an excellent research ground in the area of cardiovascular and respiratory medicine. The kinetics of changes in physiological responses during incremental exercise have previously been studied in many cardiorespiratory conditions such as asthma, obstructive and restrictive lung disorders, hypertension, peripheral vascular disease and cardiac dysfunction (I2). One of the areas of research which extensively utilizes cardiopulmonary exercise testing as an assessment tool is heart failure. The cardiopulmonary exercise test facilitates the understanding of exercise pathophysiology in heart failure, in order to construct 
appropriate treatment regime and rehabilitation program for the patients.

It has been documented that patients with congestive heart failure have a peak oxygen consumption that is generally half (range of eight to $21 \mathrm{~mL} / \mathrm{kg} / \mathrm{min}$ ) of that observed in age-matched healthy subjects (13). Although it has been a common believe that physical activity is predominantly limited by the reduction in cardiac output, peripheral factors (impaired vasodilatation and muscle deconditioning) are now recognized as major concomitant contributors to exercise intolerance in heart failure patients. It has been shown that patients with congestive heart failure have generalized skeletal muscle atrophy, most notably in type II ('fastswitch') fibres, which contribute to diminution of exercise capacity (14). In a recent study which examined the relationship between myocardial dysfunction and peripheral haemodynamic factors of exercise intolerance in heart failure, it was found that in patients with mild-to-moderate heart failure, peak $\mathrm{VO}_{2}$ significantly correlated with central haemodynamic factor (cardiac index and right atrial pressure), whereas in severe heart failure, peripheral factors (forearm blood flow, vascular resistance and venous tone) assumed greater importance in determining exercise capacity (I5). The shift from central to peripheral haemodynamic factors limiting physical exertion in heart failure was suggestive of the increasing importance of peripheral component as exercise-limiting factor, in parallel to the progression of the disease.

Neurohormonal activation has been associated with limitation in exercise capacity in heart failure patients. Brain Naturetic Peptide (BNP) has recently been found to have a role in identifying chronic heart failure patients with moderate to severe impairment in exercise capacity. A recent study revealed significant correlation between BNP levels with oxygen uptake. A BNP above $316 \mathrm{pg} / \mathrm{ml}$ was associated with a risk ratio of 6.8 for a reduced exercise capacity with a peak $\mathrm{VO}_{2}$ of below $14 \mathrm{ml} / \mathrm{min} / \mathrm{kg}$ (I6).

Cardiopulmonary exercise test is also a valuable research tool for assessing therapeutic interventions in heart failure. Treatment with Alacepril (long-acting sulphydryl-containing angiotensin-converting enzyme inhibitor) has been shown to improve functional status and exercise capacity in patients with mild-to-moderate chronic heart failure (17). In another recent study on pharmacological treatment for heart failure, 106 ambulatory heart failure patients were randomized to either spironolactone therapy ( 12.5 to $50 \mathrm{mg} /$ day) or control group (18). Peak oxygen consumption was found to be significantly increased in patients treated with the highest administrated dose $(50 \mathrm{mg})$ of spironolactone $(17.7 \pm 5.2$ vs. $18.5 \pm 5.9, p=0.01)$ and decreased in control group $(19.1 \pm 5.6$ vs. $17.9 \pm 5.3)$ after twelve months of treatment. The data obtained from cardiopulmonary exercise test supported the use of spironolactone adjunctive to other novel pharmacological therapy, to optimize the clinical condition and prognosis of patients with chronic heart failure.

Anemia is common in elderly patients with chronic heart failure. An increase in haemoglobin level could enhance exercise performance by increasing oxygen delivery. In a recent randomized single blind prospective study (19), treatment with erythropoietin (EPO) for three months was shown to result in significant improvement of peak $\mathrm{VO}_{2}(\mathrm{I} I .0 \pm 1.8$ to $12.7 \pm 2.8$ $\mathrm{ml} / \mathrm{kg} / \mathrm{min}, p<0.05)$ and exercise duration $(590 \pm 107$ to $657 \pm$ II $9 \mathrm{sec}, p<0.004)$ in patients with moderate to severe heart failure.

The research utility of cardiopulmonary exercise testing continues to expand in this recent era of evidencebased medicine. Cardiopulmonary exercise test is now utilized in virtually any domain of research that requires objective assessment of the subject's fitness level, particularly, the cardiovascular and pulmonary functional capacity, discrimination of the etiologic factors of exercise intolerance, evaluation of therapy, as well as for prognostic indication. The large body of data obtained from research utility of cardiopulmonary exercise test forms the basis of its application in the clinical practice.

\section{Utility of cardiopulmonary exercise testing in clinical practice}

Because of the important nature of the physiological profiles obtained during the cardiopulmonary exercise test, it has now been widely applied in a wide spectrum of clinical setting, including Respiratory Medicine, Cardiology, Sports Medicine, Occupational and Rehabilitative Medicine. It has a value in facilitating the clinicians in many different levels of clinical decision-making, including diagnosis, assessment of severity and progression of disease, prognosis and response to treatment. However, the interpretation of test results should be on an individual basis, after consideration of the patient's full clinical history, physical examination and other diagnostic modalities.

\section{Clinical application of cardiopulmonary exercise test in respiratory medicine}

Cardiopulmonary exercise test is clinically useful to objectively determine exercise capacity and establish exercise limitations in patients with lung disease. It helps to characterize limitations of breathing mechanics, peripheral deconditioning and gas exchange disorders, in the presence of restrictive and obstructive pulmonary diseases. Many patients with pulmonary disorders 
also have concomitant disease of other organ systems that may affect exercise performance. Cardiopulmonary exercise test also helps to distinguish and identify other contributing factors of exercise intolerance in these patients.

Patients with chronic obstructive pulmonary disease may complain of gradual increase in exertional dyspnoea over time, which may be multi-factorial (ventilatory inefficiency, cardiovascular limitation, muscle deconditioning and psychological problem). The study of ventilatory reserve and breathing pattern during cardiopulmonary exercise test helps to objectively define the dominant cause of unexplained dyspnoea, especially when exertional symptoms are disproportionate to resting pulmonary function test (20). Patients with ventilatory-related exertional dyspnoea fail to reach anaerobic threshold or achieve $\mathrm{VO}_{2}$ max. They use more than $70 \%$ of their maximum voluntary ventilation at peak exercise and develop arterial desaturation with exercise. In contrast, cardiac patients with dyspnoea on exertion are able to attain $\mathrm{VO}_{2}$ max and anaerobic threshold, use less than $50 \%$ of their maximum breathing effort and do not develop desaturation during physical exertion (I).

In interstitial lung disease and pulmonary vascular disease, cardiopulmonary exercise test aid in early detection of subtle pulmonary gas exchange abnormalities, not revealed by routine testing. It also permits physiologic monitoring of severity of the illness and therapeutic intervention. Cardiopulmonary test also helps to quantify and qualify the response to oxygen therapy and pulmonary rehabilitation, as well as facilitate in the monitoring of disease progression in patients with chronic pulmonary disorders.

Determination of preoperative pulmonary function is crucial in avoiding complications from pulmonary resection surgery. Risk for perioperative complications can generally be stratified by the level of peak oxygen consumption $\left(\mathrm{VO}_{2} \mathrm{max}\right)$ obtained from cardiopulmonary exercise test (2I). Generally, patients with preoperative $\mathrm{VO}_{2}$ max more than $20 \mathrm{~mL} / \mathrm{kg} / \mathrm{min}$ are not at increased risk of complications or death. $\mathrm{VO}_{2}$ max less than $15 \mathrm{~mL} / \mathrm{kg} / \mathrm{min}$ indicates an increase risk of perioperative complications, whereas patients with $\mathrm{VO}_{2}$ max less than $10 \mathrm{~mL} / \mathrm{kg} / \mathrm{min}$ have a high risk for post-operative complications. Desaturation during exercise test has also been associated with an increased perioperative complication.

\section{Clinical application of cardiopulmonary exercise testing in cardiovascular diseases}

Cardiopulmonary exercise has been clinically utilized in the evaluation of patients with various forms of cardiovascular diseases, to objectively determine func- tional capacity and prognostic outcome, as well as to assess response to therapeutic interventions. Submaximal exercise testing is routinely performed in patients after an acute coronary event before hospital discharge to evaluate their functional capacity and predict future adverse cardiac events. In a recent study (22) involving 740 men with unstable angina or non-Q wave myocardial infarction, who underwent predischarge cycle ergometer exercise testing, the major predictors of one year infarction-free survival, were found to be the total number of leads with ischaemic ST-segment depression and peak workload attained during the cardiopulmonary exercise testing.

Cardiopulmonary exercise testing also offers an objective evaluation of functional capacity in patients with heart failure. Peak $\mathrm{VO}_{2}$ provides important prognostic information in patients with chronic heart failure. Previous study has demonstrated a $77 \%$ oneyear mortality rate for patients with peak $\mathrm{VO}_{2}$ less than $10 \mathrm{~mL} / \mathrm{kg} / \mathrm{min}$ and a $21 \%$ mortality rate for those with $\mathrm{VO}_{2}$ between 10 to $18 \mathrm{ml} / \mathrm{kg} / \mathrm{min}$ (23). The value of peak exercise oxygen consumption in determining the optimal timing of cardiac transplantation has been established by a later study by others who performed cardiopulmonary stress test on 116 ambulatory heart failure patients referred for cardiac transplantation (24). The patients were divided into three groups based on peak oxygen consumption $\left(\mathrm{VO}_{2} \max \right)$ cut-off point of $14 \mathrm{~mL} / \mathrm{kg} / \mathrm{min}$. Group I consisted of 35 accepted candidates for transplant, who achieved $\mathrm{VO}_{2}$ max of less than $14 \mathrm{~mL} / \mathrm{kg} / \mathrm{min}$. Group 2 included 52 patients with $\mathrm{VO}_{2}$ max more than $14 \mathrm{~mL} / \mathrm{kg} / \mathrm{min}$, who had transplant deferred. Group 3 involved 27 heart failure patients with $\mathrm{VO}_{2}$ max less than 14 $\mathrm{mL} / \mathrm{kg} / \mathrm{min}$ but had a significant co-morbidity that precluded the decision for transplantation. The study revealed a good prognosis (one-year survival rate of 94\%) in Group I patients, who obtained $\mathrm{VO}_{2}$ max more than $14 \mathrm{~mL} / \mathrm{kg} / \mathrm{min}$. On the other hand, accepted transplant candidates with $\mathrm{VO}_{2}$ max less than 14 $\mathrm{mL} / \mathrm{kg} / \mathrm{min}$ had a one-year survival rate of $70 \%$, whereas patients in Group 3 with low $\mathrm{VO}_{2}$ max and significant co-morbidity had a $47 \%$ one year survival rate. A more recent prognostic study (25) involving 154 heart failure transplant candidates with peak $\mathrm{VO}_{2}$ less or equal to $14 \mathrm{~mL} / \mathrm{kg} / \mathrm{min}$ revealed a threeyear survival rate of $55 \%$ in patients $(n=77)$ who were unable to reach peak exercise systolic blood pressure of $120 \mathrm{mmHg}$, compared to $83 \%$ three-year survival rate in patients $(n=74)$ who accomplished this peak exercise systolic blood pressure cut-off point. Multivariate analysis revealed that peak exercise systolic blood pressure $(p=0.0005)$ and per cent predicted $\mathrm{VO}_{2}$ less or equal to $50 \%(p=0.04)$ were the two most important variables for estimating prognostic risk. The data from these studies formed the basis of 
the clinical application of cardiopulmonary stress testing for prognostic stratification and selection of potential candidates for heart transplant, as well as identifying nominated candidates whose transplant could be safely deferred in the event of limited resources.

Cardiopulmonary exercise test can be used to objectively monitor progression of disease and evaluate the effect of treatment on cardiac patient's functional capacity. Serial measurements of peak $\mathrm{VO}_{2}$ have been used to determine the effect of electrical cardioversion on functional capacity of patients with chronic atrial fibrillation. A previous study concluded that the restoration of sinus rhythm was associated with a delayed improvement in exercise capacity (28 days post-cardioversion) (26), which may in part be due to a slow improvement in atrial contractility and peak cardiac output after electrical cardioversion. Maintenance of sinus rhythm following electrical cardioversion resulted in sustained improvement of functional capacity, which is reflected by the value of peak $\mathrm{VO}_{2}(27)$.

Recently, the cardiopulmonary exercise test has also been utilized to evaluate the effect of both medical and non-pharmacological approach to management of patients with heart failure. A recent small randomized control trial evaluating the effect with beta-adrenergic blocking agent (Carvedilol) in patients with idiopathic dilated cardiomyopathy revealed improvement of submaximal exercise tolerance and cardiac haemodynamic indices following short ( 12 hours) and long-term (four months) administration of the drug (starting dose of $6.25 \mathrm{mg}$ a day with weekly increments up to maximum of $25 \mathrm{mg}$ twice a day), as compared to placebo (28). Recently, pacing therapy in heart failure has been widely accepted as part of the integrated management of advanced heart failure patients who are resistant to medical treatment. Cardiopulmonary exercise test has been routinely performed to evaluate the effect of pacing treatment in heart failure. The recent studies $($ I I,29,30) have consistently shown that cardiac resynchronization therapy (biventricular pacing) improved exercise capacity, cardiac function and quality of life in symptomatic patients with severe chronic heart failure and ventricular conduction disturbances, who are non-responsive to pharmacologic therapy.

\section{Application of cardiopulmonary exercise testing in sports medicine}

As cardiopulmonary exercise testing measures an individual's physiological responses to exercise, it is often utilized to provide an objective evaluation of exercise performance in both the healthy and symptomatic subjects, in the field of sports medicine. The results obtained from the test are essential for constructing the appropriate training regimes for athletes and rehabilitative patients. Cardiopulmonary exercise test has been previously used to assist in confirming and elucidating different etiological factors of exercise limitations in debilitating conditions such as chronic fatigue syndrome $(3 \mathrm{I})$ and in $\mathrm{HIV}+$ individuals (32), for recommendation and optimization of their exercise training program. In the athlete population, cardiopulmonary exercise can distinguish between physiologic from pathologic hypertrophy of the left ventricle (33). Serial measurements of physiologic parameters obtained from the test can also objectively evaluate fitness progress and maximize athlete's performance, particularly in endurance sports such as marathon running, swimming, cycling and rowing.

\section{Applications of cardiopulmonary exercise test in occupational and rehabilitative medicine \\ Cardiopulmonary exercise test is of great importance in the evaluation of disability and impairment. It provides an objective evaluation of exertion ability, beyond the assessment of patient's clinical history and examination findings. Apart from objective assessment of exercise capacity, cardiopulmonary exercise test also identifies exercise-induced arrhythmias, arterial desaturation and the timing of metabolic acidosis, all of which may contribute to exercise intolerance in patients with cardiopulmonary diseases. It provides objective and accurate evidence for occupational- related disability in patients who file workers' com- pensation claim.}

Exercise prescription during cardiac rehabilitation is usually based on the documentation of patient's functional capacity, cardiac and haemodynamic responses, as well as signs and symptoms associated with exertion, obtained from the cardiopulmonary stress test (34). Serial measurements of peak $\mathrm{VO}_{2}$ and ventilatory parameters are also useful to evaluate and optimize the safety and intensity of training, as well as to assess the therapeutic response to physical training in patients with cardiopulmonary diseases. Previous randomized controlled trial assessing the benefit of exercise training in patients with heart failure revealed varying degree of improvement of peak $\mathrm{VO}_{2}$ from $16 \%$ (35) to $31 \%$ (36) after six months of moderate physical training. Pulmonary rehabilitation has been proven to improve quality of life and exercise capacity in clinically stable patients with chronic obstructive pulmonary disease (37). Patients with severe chronic obstructive pulmonary disease required rehabilitation program of at least six month duration to show significant benefit over the control group. Improvement of exercise tolerance following pulmonary rehabilitation in these patients is believed to be attributed to the improvement in neuromuscular coordination and desensitization in the perception of dyspnoea. 


\section{Role of cardiopulmonary exercise testing for pre- operative evaluation of the elderly}

Major surgery is associated with high post-operative oxygen consumption and cardiac output requirement. Elderly patients may not have the ability to respond to the increased needs of surgery and trauma, due to the natural tendency for cardiac function to deteriorate with age. These patients may die from cardiac failure post-operatively, when oxygen delivery is insufficient to meet the oxygen requirement of the organs in the body. Cardiopulmonary exercise test is thus a reliable technique for pre-operative evaluation of the ability of the cardio-respiratory system to respond to the stress of surgical trauma. In a previous prognostic study (38), post-operative cardiovascular mortality was associated with oxygen uptake at anaerobic threshold $\left(\mathrm{VO}_{2}\right.$-AT) of less than $1 \mathrm{l} \mathrm{mL} / \mathrm{kg} / \mathrm{min}$. The investigator also found that an $\mathrm{VO}_{2}$-AT of less than II $\mathrm{mL} / \mathrm{kg} / \mathrm{min}$ associated with angina or ECG evidence of ischaemia during exercise, resulted in approximately $40 \%$ mortality following major surgery in patients over 60 years of age. Elderly patients who exhibited neither cardiac failure nor ischaemia based on the exercise criteria had a mortality of less than one per cent.

\section{Conclusion}

Evaluation of exercise capacity involves assessment of the interaction and adaptation of various human physiological systems to physical activity. This can be achieved by a non-invasive exercise test with integrated gas exchange analysis. The cardiopulmonary exercise test provides comprehensive assessment of functional capacity, particularly, the cardio-respiratory fitness. It can be utilized in virtually any field of research that requires objective evaluation of the subject's cardiopulmonary as well metabolic function during exercise. The research data on utility of cardiopulmonary exercise test in various clinical conditions forms the basis of its application in the clinical field. Clinicians from various specialties can now utilize the cardiopulmonary exercise test as an integral part of a patient's overall clinical evaluation. Cardiologist and respiratory physician may use the test to further understand the factors which limit exercise capacity in patients with cardiopulmonary disorders, as well as to facilitate in distinguishing between cardiac or pulmonary cause of exertional dyspnoea. Cardiopulmonary exercise test also has a clinical role in determining the optimal time for heart or lung transplant, predicting prognosis and objectively monitor disease progression and response to therapeutic interventions. In sports medicine, it is often use as an assessment tool to monitor progress of exercise training program for further optimization of exercise performance in both the athlete population as well as chronically ill patients. In the field of occupational medicine, cardiopulmonary exercise test has a great importance in objectively determining disability and impairment for workrelated compensation claims. Cardiopulmonary exercise test is now increasingly used in preoperative evaluation of patients undergoing major surgery due to its reliability in predicting peri-operative risk of morbidity and mortality. Regardless of the conditions in which the cardiopulmonary exercise test is applied to, the value of information obtained from it will only be evident when interpreted on an individual basis, after the consideration of patient's full clinical profile.

\section{References}

I. Myers JN. The physiology behind exercise testing. Prim Care 200I; 28(I):5-27.

2. Myers F. Special methods: Ventilatory Gas Exchange. In: Exercise and the Heart; Saunders 4th edition 2000; 39-58.

3. American Thoracic Society/American College of Chest Physician. ATS/ACCP Statement on Cardiopulmonary Exercise Testing. Am J Respir Crit Care Med 2003; 167:211-277.

4. MitlehnerW. Cardiopulmonary Exercise Testing: Practical guideline. VIASYS info Special Edition CPET 2002; I2-I8.

5. Miyamura M, Honda Y. Oxygen intake and cardiac output during treadmill \& bicycle exercise. J Appl Physiol 1972; 32: $185-188$.

6. Bruce RA. Exercise testing of patient with coronary heart disease. Ann Clin Res 197I; 3:323-330.

7. Myers J, Buchanan N,Walsh D et al. Comparison of the ramp vs. standard exercise protocols. J Am Coll Cardiol 1991; 17:1334-42.

8. Myers J, Bellin D. Ramp exercise protocols for clinical and cardiopulmonary exercise testing. Sports Med 2000; 30(I):23-29.

9. Cahalin LP, Semigran MJ, Dec GW. Assessment of oxygen uptake during the 6-minute walk test. Chest 1997; I I : | 465-6.

10. Auricchio A, Stellbrink C, Sack S et al. The pacing therapies for congestive heart failure (PATH-CHF) study (abstract) Am J Cardiol 1999; 83:I30D-I35D.

II. Cazeau S, Laclereq C, Lavergne T, et al for Multisite Stimulation on Cardiomyopathies (MUSTIC) Study Investigators. Effect of multisite biventricular pacing in patients with heart failure and interventricular conduction disturbances. N Engl J Med 200 I; 344: 873-880.

12. Vibhuti N, Singh. The role of gas analysis with exercise testing. Prim Care 200I; 28(I):I59-178. 
13. Brubaker, PH. Exercise intolerance in congestive heart failure: A lesson in exercise physiology. J Cardiopulm Rehabil 1997; 17(4):217-221.

14. Wilson JR, Mancini DM. Factors contributing to exercise limitation in heart failure. J Am Coll Cardiol 1993; 22:93A- 98A.

15. Florea VG, Mareyer VY, Archilov AA, et al. Central and peripheral components of chronic heart failure: Determinants of exercise intolerance. Int J Cardiol 1999; $70(1): 51-6$

16. Myers S, Graft J, Kunz D, et al. Brain naturetic peptide levels predict functional capacity in patients with chronic heart failure. J Am Coll Cardiol 2002; 40(4):718-22.

17. Kinugawa T, Osaki S, Kato M, et al. Effect of angiotensinconverting enzyme inhibitor alacepril on exercise capacity and neurohormonal factors in patients with mild to moderate heart failure. Clin Exp Pharmacol Physiol 2002; 12:1060-1065.

18. Cicoira M, Zanolla L, Rossi A, et al. Long-term, dosedependent effects of spironolactone on left ventricular function and exercise tolerance in patients with chronic heart failure. J Am Coll Cardiol 2002; 40(2):304-I0.

19. Mancini DM, Katz SD, Lang CC, et al. The effect of erythropoietin on exercise capacity in patients with moderate to severe chronic heart failure. Circulation 2003; 107(2):294-9.

20. Steven K, Mohnssen, Idelle MW, et al. Heart or Lung Disease: Determining the primary cause for dyspnoea on exertion. Chest 1998; 113:1705-10.

2I. Beckles MA, Spiro SG, Colice GL, et al.American College of Chest Physician. The physiologic evaluation of patients with lung cancer being considered for resectional surgery. Chest (Suppl I) 2003; I23:I05S-II4S.

22. Nyman I, Larsson $H$, Areskog M, et al. The predictive value of silent ischaemia on an exercise test before discharge after an episode of unstable coronary artery disease: RISC study group. Am Heart J 1992; 123:32433 I.

23. Szlahcic J, Massio B, Kramer B, et al. Correlations and prognostic implication of exercise capacity in chronic heart failure. Am J Cardiol 1985; 55: 1037-1042.

24. Mancini DM, Eisen $\mathrm{H}$, Kussumaul W, et al. Value of peak exercise oxygen consumption for optimal timing of cardiac transplant in ambulatory patients with heart failure. Circulation 1991; 83:778-786.

25. Osada N, Chaitman BR, Miller LW, et al. Cardiopulmonary exercise testing identifies low risk patients with heart failure and severely impaired exercise capacity considered for heart transplant. J Am Coll Cardiol 1998; 3 I (3):577-82.
26. Lipkin DP, Frenneaux M, Stewart R, et al. Delayed improvement in exercise capacity after cardioversion of atrial fibrillation to sinus rhythm. Br Heart J 1988; 59:572-7.

27. Gosselink ATM, Bulsma EBM, Landsman LJ, et al. Longterm effect of cardioversion on peak oxygen consumption in chronic atrial fibrillation: a two year follow-up. Eur Heart J 1994; I5: 1368-1372.

28. Metra M, Nardi M, Giubbini R, et al. Effect of short and long-term carvedilol administration on rest and exercise haemodynamic variables, exercise capacity and clinical conditions in patients with idiopathic dilated cardiomyopathy. J Am Coll Cardiol 1994; 24(7): 1678-87.

29. Grass D, Cazeau S, Ritter P, et al. Long-term results of cardiac resynchronization for heart failure patients: The Insync Clinical Trial. Circulation (Suppl) 1999; 100:510515.

30. Abraham WT, Fisher MG, Smith AL, et al for Multicenter Insync Randomized Clinical Evaluation (MIRACLE) Investigators and Coordinators. Double blind controlled trial of cardiac resynchronization in chronic heart failure. N Engl J Med 2002; 346: 1845- 1853.

31. Inbar O, Dlin R, Rotstein A, et al. Physiological responses to incremental exercise in patients with chronic fatigue syndrome. Med Sci Sports Exerc 200 I; 33(9): I463- 1470.

32. Stringer W. Mechanism of exercise limitation in HIV+ individuals (review). Med Sci Sports Exerc (Suppl) 2000; 32(7):S4I 2-42I.

33. Sharma S, Elliot PM, Whyte G, et al. Utility of metabolic exercise testing in distinguishing hypertrophic cardiomyopathy from physiologic left ventricular hypertrophy in athletes. J Am Coll Cardiol 2000; 34(3):864-70.

34. William MA. Exercise testing in cardiac rehabilitation: Exercise prescription and beyond. Cardiol Clin 200I; 19(3):4|5-3|.

35. Keteyian SJ, Levine AB, Brawner, et al. Exercise training in patients with heart failure: $A$ randomized controlled trial. Ann Int Med 1996; I24(I2): 105 I-1057.

36. Hambrecht R, Niebauer J, Fiehn E, et al. Physical training in patients with stable chronic heart failure: effects in cardiorespiratory fitness and ultrastructural abnormalities of leg muscles. J Am Coll Cardiol 1996; 25:1239-49.

37. Salman GF, Mosier MC, Beasley BW, et al. Rehabilitation for patients with chronic obstructive pulmonary disease: Meta-analysis of randomized controlled trials. J Gen Int Med 2003; 18(3):213-221.

38. Wasserman K. Preoperative evaluation of cardiovascular reserve in the elderly. Chest. 1993 Sep; 104(3): 663-4. 